a number of major and minor elements, changes in leaf outline are usually slight, the more notable being those accompanying zine deficiency.

$$
\text { M. Greenwood }
$$$$
\text { A. F. Posnette }
$$

Department of Agriculture and

West African Cacao Research Institute, Tafo, Gold Coast. Jan. 22.

${ }^{1}$ Posnette, A. F., Trop. Agric., Trinidad, 5, 87 (1941).

2 Posnette, A. F., Trop. Agric., Trinidad, 10, 188 (1945).

'Reeve, E., and Shive, J. W., Soil Sci., 57, 1, Tab. 1 (1944).

'Harder, R., and Meyer, G., Nach. Akad. Wiss. Göttingen, Math. Naturvo. Kl. (1945).

'Harder, R., and Gummer, G., Jb. wiss. Bot., 91, 359 (1941).

\section{Mechanism of Hydrogen Peroxide Formation by Spermatozoa and the Role of Amino- Acids in Sperm Motility}

IT has been demonstrated ${ }^{1}$, using freshly ejaculated bovine semen diluted with egg-yolk medium, that spermatozoa produce under aerobic conditions an inhibitor of sperm respiration. The inhibitor was afterwards identified chemically ${ }^{2}$ as hydrogen peroxide, and was shown to be formed as the result of metabolic activity of spermatozoa upon a substance present in the egg-yolk and its dialysable portion. Since then, further purification and characterization of the egg-yolk substance have been carried out; and it has now been demonstrated that hydrogen peroxide is formed as the result of an oxidative deamination by spermatozoa of the substance isolated, which is replaceable, with the same effect, only by $l$-tryptophane, $l$-phenylalanine and $l$-tyrosine, but not by any of many other amino-acids tested. The inhibition which develops as the result of the above reaction can be detected not only by its effect upon oxygen consumption but also by its effect upon the reduction of motility of spermatozoa.

The isolation of the substance, in presence of which spermatozoa produce hydrogen peroxide, was effected by dialysis of egg-yolk, followed by successive precipitations and/or extractions with organic solvents of the dialysable portion or the fractions separated therefrom. The isolated substance is easily dialysable, and soluble in water, 90 per cent ethanol and isopropanol. It is not fermentable by baker's yeast. Quantitative analysis revealed that it contains $10 \cdot 7$ per cent nitrogen, $4 \cdot 2$ per cent ash and $0 \cdot 13$ per cent sulphur (Weiler and Strauss), no phosphorus nor fermentable reducing substances. In low concentration it gives strong reactions for tryptophane and tyrosine. Only traces of glucosamine were detected before or after acid hydrolysis.

Washed suspensions of living spermatozoa of the bull, in the presence of the isolated substance, produced aerobically ammonia and hydrogen peroxide; under anaerobic conditions, however, there was neither ammonia nor hydrogen peroxide formation, and subsequent respiration and motility were unaffected. The ash of the substance had no appreciable effect on either respiration or motility. Spermatozoa inactivated by heat in presence of the substance and molecular oxygen produced neither ammonia nor hydrogen peroxide.

In view of the properties of the substance isolated, natural amino-acids were next tested individually as substrates for the metabolism and motility of bovine spermatozoa. This examination revealed that formation of ammonia and hydrogen peroxide, and subsequent inhibition of sperm respiration and motility, took place aerobically in the presence of only $l$-tryptophane, $l$-phenylalanine and $l$-tyrosine. There was neither ammonia nor hydrogen peroxide formation in the absence of oxygen or with heat-inactivated spermatozoa. The inhibition of sperm respiration and motility in presence of these substances was completely eliminated with added catalase (more than $10 \mu \mathrm{gm} . / 4 \mathrm{ml}$. sperm suspension). In the presence of $d l$-alanine, $d l$-serine, $l$-cysteine, $d l$-valine, $l$-leucine, $l$-arginine, $l$-lysine, $l$-hystidine, $l$-aspartic acid and l-glutamic acid, there was no inhibition of either sperm respiration or motility, and no formation of ammonia or hydrogen peroxide.

These results suggest that the reaction by which hydrogen peroxide is formed by living spermatozoa is an enzymic dehydrogenation and deamination proceeding in the presence of molecular oxygen as hydrogen acceptor as follows :

R. $\mathrm{CH}_{2} \cdot \mathrm{CH}\left(\mathrm{NH}_{2}\right) \cdot \mathrm{COOH}+\mathrm{O}_{2}+\mathrm{H}_{2} \mathrm{O} \longrightarrow$

R. $\mathrm{CH}_{2} \cdot \mathrm{CO} \cdot \mathrm{COOH}+\mathrm{H}_{2} \mathrm{O}_{2}+\mathrm{NH}_{3}$,

where $R$ can be only phenyl, $p$-hydroxyphenyl or indolyl groups, but none of the other groups tested. The $l$-amino-acid oxidase (or oxidases) of living bovine spermatozoa, which affects only the three aromatic amino-acids, appears to be, therefore, more specific than the $l$-amino-acid oxidase present in some other animal tissues, which can utilize as substrates a much larger range of $l$-amino-acids ${ }^{3,4}$.

These findings may have an important bearing on the fertilizing capacity of spermatozoa, and on their storage and survival in storage media, such as eggyolk, and in natural secretions, especially in view of the fact that $l$-tryptophane, $l$-phenylalanine and $l$-tyrosine are widely distributed in animal tissues and body fluids. Moreover, the spermatozoa have an exceptionally low capacity for decomposing hydrogen peroxide; and hydrogen peroxide, even in very low concentrations which have not so far been chemically detectable, has a detrimental effect on the respiratory activity and motility of spermatozoa, as demonstrated by the elimination of these effects in presence of added catalase.

This work, carried out on behalf of the Agricultural Research Council, will be published in detail elsewhere.

School of Agriculture, J. Tosic

University of Cambridge. Feb. 20.

${ }^{1}$ Tosic, J., and Walton, A., Nature, 156, 507 (1945).

sosic, J., and Walton, A., Nature, 158, 485 (1946).

${ }^{8}$ Krebs, H. A., Z. physiol. Chem., 216, 204 (1933) ; Biochem. J., 29, 1620 (1935).

- Blanchard, M., et al., J. Biol. Chem., 155, 421 (1944).

\section{The Water-balance Principle of Crustacean Eye-stalk Extracts}

IT has long been known ${ }^{1}$ that extracts of erustacean eye-stalks contain a principle which not only influences the colour changes of crustaceans but also induces expansion of the melanophores in amphibians, acting in this respect like the intermediate lobe pituitary hormone. It was therefore of considerable interest when Gray and Ford ${ }^{2}$ reported that extracts of crustacean eye-stalks produce an increase of water uptake in frogs similar to that observed after the injection of neurohypophysial extracts. No structure homologous to the vertebrate neurohypophysis has so far been identified in invertebrates; but the results of the American authors suggest the possibility that crustaceans, although they have no pituitary gland, elaborate pituitary-like hormones. 